\title{
Pseudo-Cryptanalysis of Luffa
}

\author{
Keting Jia ${ }^{1,2}$, Yvo Desmedt ${ }^{3}$, Lidong Han ${ }^{1}$, Xiaoyun Wang ${ }^{1,2 \star}$ \\ 1 Key Laboratory of Cryptologic Technology and Information Security, Ministry of Education, \\ Shandong University, China \\ \{ktjia, hanlidong\}@mail.sdu.edu.cn \\ 2 Institute for Advanced Study, Tsinghua University, China \\ xiaoyunwang@mail.tsinghua.edu.cn \\ 3 Department of Computer Science, University College London, UK \\ y.desmedtecs.ucl.ac.uk
}

\begin{abstract}
In this paper, we present the pseudo-collision, pseudo-second-preimage and pseudo-preimage attacks on the SHA-3 candidate algorithm Luffa. The pseudocollisions and pseudo-second-preimages can be found easily by computing the inverse of the message injection function at the beginning of Luffa. We explain in details the pseudo-preimage attacks. For Luffa-224/256, given the hash value, only 2 iteration computations are needed to get a pseudo-preimage. For Luffa384 , finding a pseudo-preimage needs about $2^{64}$ iteration computations with $2^{67}$ bytes memory by the extended generalized birthday attack. For Luffa-512, the complexity is $2^{128}$ iteration computations with $2^{132}$ bytes memory.

It is noted that, we can find the pseudo-collision pairs and the pseudo-second images only changing a few different bits of initial values. That is directly converted to the forgery attack on NMAC in related key cases.
\end{abstract}

Key words: Luffa, pseudo-collision, pseudo-second-preimage, pseudo-preimage, generalized birthday attack

\section{Introduction}

A cryptographic hash function is defined as a function that computes a fixed size message digest from arbitrary size messages. It has been widely used as a fundamental primitive in many cryptographic schemes and protocols, such as electronic signature, authentication of messages, electronic commerce and bit commitment, etc. In the past years, the cryptanalysis of hash functions has achieved tremendous progress with the construction of collisions. In particular, Wang et al. proposed new techniques to find efficiently collisions on the main hash functions from the MD4 family (e.g., MD4 [8], RIPEMD [8], MD5 [11], SHA-0 [9] and SHA-1 [10]). Moreover the techniques can be applied to explore the second-preimage of MD4 [12], forgery and partial key-recovery attacks on HMAC and NMAC [34]. Kelsey and Schneier [5] provided a second preimage attack on the iterated hash functions with Merkle-Damgård strengthening, which shows a vulnerability of the Merkle-Damgård construction. Responding to advances in the cryptanalysis of hash functions, NIST held two hash workshops to evaluate the

\footnotetext{
* Corresponding author
} 
security of its approved hash functions and to solicit public comments on its cryptographic hash function policy and standard. Finally, NIST opened a public competition to develop a new hash function called "SHA-3", similar to the development process of the Advanced Encryption Standard (AES). There are 64 new proposals for hash functions have been submitted to the SHA-3 project, of which 51 submissions have come into the first round. In July, 2009, NIST has selected 14 second round candidates of the SHA-3. Luffa [2] is one of them, proposed by De Cannière, Sato and Watanabe.

In this paper, we give some cryptanalytic results of Luffa with free initial values. The pseudo-collision and pseudo-second-preimage can be obtained easily by the message injection function of Luffa, which only changes a few bits of the initial values. This paper shows a pseudo-collision and pseudo-second-preimage example for Luffa256 and gives the actual attacks. For Luffa-224/256, only 2 iteration computations are needed to get the pseudo-preimage. A pseudo preimage example for Luffa-256 is shown in this paper, which only changes 2 256-bit words of the initial values with 3 256-bit words. We use the extended generalized birthday attack [7] to compute the pseudopreimage of Luffa- 384 with $2^{64}$ iteration computations and $2^{64}$ table lookups. The time complexity and data complexity are both $2^{128}$ to get the pseudo-preimage for Luffa-512.

This paper is organized as follows. In Section 2, we list some notations and give a brief description of Luffa. Section 3 shows the pseudo-collision and pseudo-secondpreimage attacks on Luffa. The pseudo-preimage attacks for Luffa is given in Section 4. The improved pseudo-preimage attacks for Luffa-384/512 are shown in Section 5. Finally, we summarize our results in Section 6.

\section{Preliminaries and Notations}

In this section, we first list some notations used in this paper, and then give a brief description of Luffa.

\subsection{Notations}

$$
\begin{array}{cl}
X \| Y & : \text { the concatenation of two messages } X \text { and } Y . \\
h_{w}(X) & : \text { the } w \text { most significant bits of } X . \\
l_{w}(X) & : \text { the } w \text { least significant bits of } X . \\
\lfloor a\rfloor & : \text { the greatest integer less than or equal to } a . \\
\left(b_{0}, b_{1}, \ldots, b_{m}\right)^{T} & : \text { the transposed matrix of }\left(b_{0}, b_{1}, \ldots, b_{m}\right), \text { where } b_{i}(1 \leq i \leq m) \text { are } \\
& \text { column vectors. } \\
a \lll j & : \text { left rotation of } a \text { by } j \text { bits. }
\end{array}
$$

\subsection{Description of Luffa}

Luffa [2], a candidate algorithm for the second round of the SHA-3, was proposed by De Cannière et al. The chaining of Luffa is a variant of a sponge function. Fig 1 depicts the basic structure. For any message, Luffa can produce the hash values with 224, 256, 384 or 512 bits, which are denoted as Luffa-224/256/384/512 respectively. The message padding method consists of appending a single bit ' 1 ' followed by the minimum bits of 
' 0 ' such that the length of the result is a multiple of 256 . Let $M=M_{0}\|\cdots\| M_{m-1}$ be a message after padding, where $M_{i}(0 \leq i<m)$ are 256-bit blocks. The iteration function of Luffa is a composition of a message injection function $M I$ and a permutation $P$ with $w$ 256-bit inputs, where $w=3,4$ or 5 for Luffa-224/256, Luffa-384 and Luffa512 respectively. The permutation $P$ includes $w$ permutations $Q_{0}, Q_{1}, \ldots, Q_{w-1}$, where $Q_{j}$ is the permutation with 256 -bit input, $j=0,1, \ldots, w-1$. Let the input of the $i-$ th iteration be $\left(H_{0}^{(i-1)}, \ldots, H_{w-1}^{(i-1)}, M_{i-1}\right)$, the $i-$ th iteration is computed as follows,

$$
\begin{aligned}
& X_{0}\|\cdots\| X_{w-1}=\operatorname{MI}\left(H_{0}^{(i-1)}, \ldots, H_{w-1}^{(i-1)}, M_{i-1}\right) \text {, } \\
& H_{j}^{(i)}=Q_{j}\left(X_{j}\right), j=0,1, \ldots, w-1,
\end{aligned}
$$

where $\left(H_{0}^{i}, \ldots, H_{w-1}^{i}\right)$ is the $i$-th iteration output, and $\left(H_{0}^{0}, \ldots, H_{w-1}^{0}\right)$ is the initial value. Final operations, called a finalization are used to the chaining value $\left(H_{0}^{(m-1)}, \ldots, H_{w-1}^{(m-1)}\right)$. For Luffa-224/256, the finalization consists of a blank iteration and a XOR operation $O F$, where the blank iteration means an iteration with a fixed message $M_{m}=\mathbb{O}$, where $\mathbb{O}$ denotes 256-bit zeros, the operation $O F$ XORs $w$ 256-bit values and outputs the result 256-bit value. For Luffa-384/512, the finalization includes two blank iterations and two XOR operations, see Fig. 11 The output of Luffa-256 is $Z_{0}$, the output of Luffa-512 is $Z_{0} \| Z_{1}$. The outputs of Luffa-224 and Luffa-384 are the truncation of the Luffa-256 and Luffa-512 respectively. Here

$$
Z_{i}=\bigoplus_{j=0}^{w-1} H_{j}^{(m+i)}, \quad i=0,1
$$

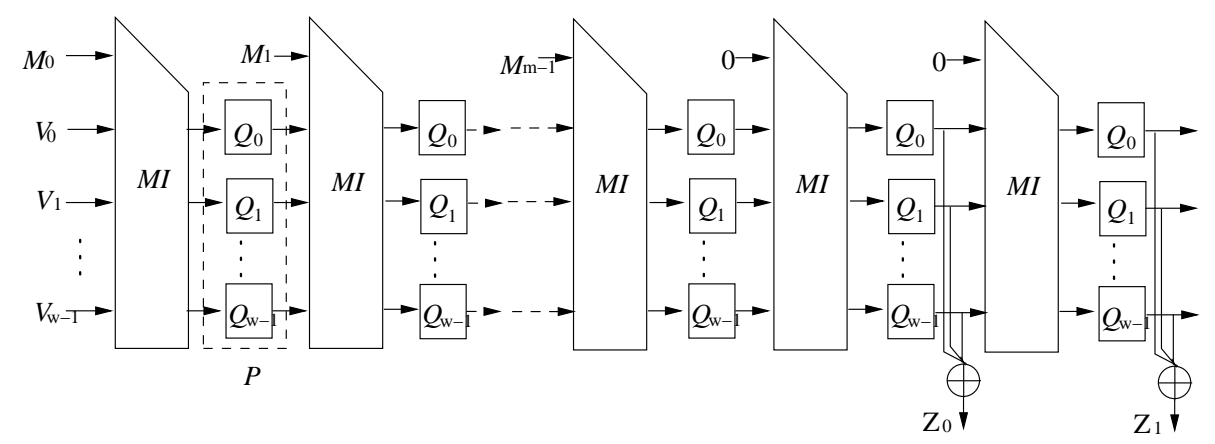

Fig. 1. The Structure of Luffa Hash Function

Message Injection Function MI The message injection functions $M I$ can be represented by the matrix over a field $G F\left(2^{8}\right)$. The multiplication over the field $G F\left(2^{8}\right)$ is modulo $\phi(x)=x^{8}+x^{4}+x^{3}+x+1$, corresponding to " $0 \mathrm{x} 11 \mathrm{~b}$ ". The map from 8 32-bit 
words $\left(h_{0}, \ldots, h_{7}\right)$ to 32 8-bit elements of the field is defined by $\left(\Sigma_{0 \leq k<8} h_{k, l} x^{k}\right)_{0 \leq l<32}$. Let $A_{w \times(w+1)}=\left(a_{0}, a_{1}, \ldots, a_{w-1}, a_{w}\right)$ represent the matrix of $M I$, where $a_{i}(0 \leq i \leq w)$ are column vectors. Then $\left(X_{0}, X_{1}, \ldots, X_{w-1}\right)^{T}=A_{w \times(w+1)} \circ\left(H_{0}, H_{1}, \ldots, H_{w-1}, M\right)^{T}$. For Luffa-224/256, w=3,

$$
A_{w \times(w+1)}=\left(\begin{array}{l}
0 \times 3,0 \times 2,0 \times 2,0 \times 1 \\
0 \times 2,0 \times 3,0 \times 2,0 \times 2 \\
0 \times 2,0 \times 2,0 \times 3,0 \times 4
\end{array}\right),
$$

where the elements $0 \mathrm{x} 1,0 \mathrm{x} 2,0 \mathrm{x} 3,0 \mathrm{x} 4$ correspond to polynomials $1, x, x+1, x^{2}$ respectively.

For Luffa-384,

$$
A_{w \times(w+1)}=\left(\begin{array}{c}
0 x 4,0 \times 6,0 \times 6,0 \times 7,0 \times 1 \\
0 x 7,0 \times 4,0 \times 6,0 \times 6,0 \times 2 \\
0 x 6,0 \times 7,0 \times 4,0 \times 6,0 \times 4 \\
0 x 6,0 x 6,0 \times 7,0 \times 4,0 \times 8
\end{array}\right) .
$$

For Luffa-512,

$$
A_{w \times(w+1)}=\left(\begin{array}{l}
0 x 0 F, 0 x 08,0 x 0 A, 0 x 0 A, 0 x 08,0 x 01 \\
0 x 08,0 x 0 F, 0 x 08,0 x 0 A, 0 x 0 A, 0 x 02 \\
0 x 0 A, 0 x 08,0 x 0 F, 0 x 08,0 x 0 A, 0 x 04 \\
0 x 0 A, 0 x 0 A, 0 x 08,0 x 0 F, 0 x 08,0 x 08 \\
0 x 08,0 x 0 A, 0 x 0 A, 0 x 08,0 x 0 F, 0 x 10
\end{array}\right) .
$$

The Permutation $Q_{j}$ The permutation $Q_{j}$ is defined as a composition of an input tweak and 8 steps. Let $a_{0}, \ldots, a_{7}$ be the 256-bit input of the $Q_{j}, b_{0}, \ldots, b_{7}$ be the output of tweak. The tweak is defined as follows:

$$
\begin{aligned}
& b_{i}=a_{i}, \text { for } 1 \leq i<4 ; \\
& b_{i}=a_{i} \lll j, \text { for } 4 \leq i<8 .
\end{aligned}
$$

After tweak, there are 8 steps in the permutation, and each step consists of the following three functions: SubCrumb, MixWord and AddConstant.

SubCrumb is defined as:

$$
\begin{aligned}
& x_{3, l}\left\|x_{2, l}\right\| x_{1, l} \| x_{0, l}=S\left(b_{3, l}\left\|b_{2, l}\right\| b_{1, l} \| b_{0, l}\right), 0 \leq l<32, \\
& x_{4, l}\left\|x_{7, l}\right\| x_{6, l} \| x_{5, l}=S\left(b_{4, l}\left\|b_{7, l}\right\| b_{6, l} \| b_{5, l}\right), 0 \leq l<32,
\end{aligned}
$$

where $S$ denotes a S-box with 4-bit input and 4-bit output. MixWord is defined as:

$$
\begin{aligned}
y_{k+4} & =x_{k+4} \oplus x_{k}, \quad y_{k}=x_{k} \lll 2, \\
y_{k} & =y_{k} \oplus y_{k+4}, \quad y_{k+4}=y_{k+4} \lll 14, \\
y_{k+4} & =y_{k+4} \oplus y_{k}, \quad y_{k}=y_{k} \lll 10, \\
y_{k} & =y_{k} \oplus y_{k+4}, \quad y_{k+4}=y_{k+4} \lll 1 .
\end{aligned}
$$

We do not give the description for AddConstant since it has no impact on our cryptanalysis. For more details about Luffa, consult [2]. 


\section{Pseudo-Collision and Pseudo-Second-Preimage Attacks on Luffa}

In this section, we give some cryptanalysis for Luffa when the initial value $I V$ is free. Flipping 5 bits of $I V$ for Luffa-256 is enough to get a pseudo-collision or pseudosecond-preimage. For Luffa-384, 7 bits of $I V$ are needed to be changed to get a pseudocollision or pseudo-second-preimage. There is a 12-bit difference in the $I V$ to get a pseudo-collision or pseudo-second-preimage for Luffa-512. This can be used to construct the related key attack for the corresponding MACs using the secret key as initial value.

For the message injection function $M I$, the input is $(w+1) 256$-bit words, and the output is $w 256$-bit words. So, it is a many-to-one function. It is easy to know that, any $w$ columns of the $M I$ matrix consists of an invertible matrix. So there are exactly $2^{256}$ inputs corresponding to any given output of $M I$. Given any $M I$ output $\left(X_{0}, X_{1}, \ldots, X_{w-1}\right)$, if one entry of $H_{0}, H_{1}, \ldots, H_{w-1}$ and $M$ is fixed, we can easily compute the solution to other entries. Any pair of inputs with the same output of $M I$ consists of a pseudocollision of Luffa, that is the output difference of $M I$ is zero.

For Luffa-224/256, the input difference is $\left(\Delta H_{0}, \Delta H_{1}, \Delta H_{2}, \Delta M\right)$, and the output difference of $M I$ is $(\mathbb{O}, \mathbb{O}, \mathbb{O})$, here $\mathbb{O}$ denotes 256 -bit zeros. They satisfy the following equations.

$$
\begin{aligned}
3 \circ \Delta H_{0} \oplus 2 \circ \Delta H_{1} \oplus 2 \circ \Delta H_{2} \oplus \Delta M & =\mathbb{0}, \\
2 \circ \Delta H_{0} \oplus 3 \circ \Delta H_{1} \oplus 2 \circ \Delta H_{2} \oplus 2 \Delta M & =\mathbb{0}, \\
2 \circ \Delta H_{0} \oplus 2 \circ \Delta H_{1} \oplus 3 \circ \Delta H_{2} \oplus 4 \Delta M & =\mathbb{0} .
\end{aligned}
$$

From the equations, it is easy to get $\Delta H_{0}=0 x f 2 \circ \Delta M, \Delta H_{1}=0 x f 1 \circ \Delta M$ and $\Delta H_{2}=$ $0 x f 7 \circ \Delta M$. Let $I V$ be the standard initial value, given a message $M$, the message $M^{\prime}=M \oplus \Delta M$, with another initial value $I V^{\prime}=I V \oplus\left(\Delta H_{0}, \Delta H_{1}, \Delta H_{2}\right)$ is the pseudopreimage of $M$, i.e. Luffa-256 $(I V, M)=$ Luffa-256 $\left(I V^{\prime}, M^{\prime}\right)$. There are only 5 bits different between $I V$ and $I V^{\prime}$, which is minimum, when the message difference $\Delta M=$ $\left(2^{i}, 2^{i}, 0,0,0,0,0,0\right),\left(0,2^{i}, 2^{i}, 0,0,0,0,0\right),\left(0,0,2^{i}, 2^{i}, 0,0,0,0\right),\left(0,0,0,2^{i}, 2^{i}, 0,0,0\right)$ or $\left(0,0,0,0,2^{i}, 2^{i}, 0,0\right)$ for $(0 \leq i<32)$.

Let the input difference be $\left(\Delta H_{0}, \Delta H_{1}, \Delta H_{2}, \Delta H_{3}, \Delta M\right)$, and the output difference of $M I$ be $(\mathbb{O}, \mathbb{O}, \mathbb{O}, \mathbb{O})$ for Luffa-384 such that

$$
\begin{aligned}
4 \circ \Delta H_{0} \oplus 6 \circ \Delta H_{1} \oplus 6 \circ \Delta H_{2} \oplus 7 \circ \Delta H_{3} \oplus \Delta M & =\mathbb{0}, \\
7 \circ \Delta H_{0} \oplus 4 \circ \Delta H_{1} \oplus 6 \circ \Delta H_{2} \oplus 6 \circ \Delta H_{3} \oplus 2 \Delta M & =\mathbb{0}, \\
6 \circ \Delta H_{0} \oplus 7 \circ \Delta H_{1} \oplus 4 \circ \Delta H_{2} \oplus 6 \circ \Delta H_{3} \oplus 4 \Delta M & =\mathbb{0}, \\
6 \circ \Delta H_{0} \oplus 6 \circ \Delta H_{1} \oplus 7 \circ \Delta H_{2} \oplus 4 \circ \Delta H_{3} \oplus 8 \Delta M & =\mathbb{0} .
\end{aligned}
$$

By the system of equations, we can deduce $\Delta H_{0}=8 \circ \Delta M, \Delta H_{1}=0 x a \circ \Delta M, \Delta H_{2}=$ $8 \circ \Delta M$ and $\Delta H_{3}=0 x f \circ \Delta M$. There is a 7-bit difference in the initial values when $\Delta M=\left(2^{i}, 0,2^{i}, 2^{i}, 0,0,0,2^{i}\right)(0 \leq i<32)$. The message $M^{\prime}=M \oplus \Delta M$ with $I V^{\prime}=I V \oplus$ $\left(\Delta H_{0}, \Delta H_{1}, \Delta H_{2}, \Delta H_{3}\right)$ is the pseudo-preimage of the given message $M$, that is to say Luffa-384 $(I V, M)=$ Luffa-384 $\left(I V^{\prime}, M^{\prime}\right)$.

Given the input difference of Luffa-512 $\left(\Delta H_{0}, \Delta H_{1}, \Delta H_{2}, \Delta H_{3}, \Delta H_{4}, \Delta M\right)$ and the output difference of $M I(\mathbb{O}, \mathbb{O}, \mathbb{O}, \mathbb{O}, \mathbb{O})$, we can compute that $\Delta H_{0}=0 x b e \circ \Delta M$, 
$\Delta H_{1}=0 \times 3 c \circ \Delta M, \Delta H_{2}=0 \times 25 \circ \Delta M, \Delta H_{3}=0 \times 17 \circ \Delta M$ and $\Delta H_{4}=0 \times 75 \circ \Delta M$ from the following equations.

$$
\begin{aligned}
0 x f \circ \Delta H_{0} \oplus 0 x 8 \circ \Delta H_{1} \oplus 0 x a \circ \Delta H_{2} \oplus 0 x a \circ \Delta H_{3} \oplus 0 x 8 \circ \Delta H_{4} \oplus \Delta M & =\mathbb{0}, \\
0 x 8 \circ \Delta H_{0} \oplus 0 x f \circ \Delta H_{1} \oplus 0 x 8 \circ \Delta H_{2} \oplus 0 x a \circ \Delta H_{3} \oplus 0 x a \circ \Delta H_{4} \oplus 0 x 2 \Delta M & =\mathbb{0}, \\
0 x a \circ \Delta H_{0} \oplus 0 x 8 \circ \Delta H_{1} \oplus 0 x f \circ \Delta H_{2} \oplus 0 x 8 \circ \Delta H_{3} \oplus 0 x a \circ \Delta H_{4} \oplus 0 x 4 \Delta M & =\mathbb{0}, \\
0 x a \circ \Delta H_{0} \oplus 0 x a \circ \Delta H_{1} \oplus 0 x 8 \circ \Delta H_{2} \oplus 0 x f \circ \Delta H_{3} \oplus 0 x 8 \circ \Delta H_{4} \oplus 0 x 8 \Delta M & =\mathbb{0}, \\
0 x 8 \circ \Delta H_{0} \oplus 0 x a \circ \Delta H_{1} \oplus 0 x a \circ \Delta H_{2} \oplus 0 x 8 \circ \Delta H_{3} \oplus 0 x f \circ \Delta H_{4} \oplus 0 x 10 \Delta M & =\mathbb{0} .
\end{aligned}
$$

When $\Delta M=\left(0,2^{i}, 2^{i}, 2^{i}, 0,2^{i}, 0,0\right),\left(2^{i}, 2^{i}, 0,0,2^{i}, 0,0,2^{i}\right)$ or $\left(0,0,2^{i}, 0,0,2^{i}, 2^{i}, 2^{i}\right)$ for $(0 \leq i<32)$, the number of bits with difference in the initial value is least, which is 12 .

Table 1 shows a pseudo-second-preimage example for the message $M_{0}=(0$ xaaaaaaaa, 0xaаaаaаaа, 0xaаaаaаaа, 0xaаaаaаaа, 0xaаaаaаaа, 0xaаaаaаaа, 0xaаaаaаaа, 0xaаaаaаaа).

From the above description, only a few bits are needed to be changed to get a collision pair or the second-preimage for a given message. It is obvious that we can directly construct the forgery attack on NMAC based on Luffa in the related key case, for the NMAC replaces the fixed $I V$ in hash function with a secret key[1]. The NMAC function, on input message $M$ and a pair of independent keys $\left(K_{1}, K_{2}\right)$, is defined as:

$$
\operatorname{NMAC}_{\left(K_{1}, K_{2}\right)}(M)=H\left(K_{1}, H\left(K_{2}, M\right)\right) \text {. }
$$

When $H$ is the Luffa hash function, a forgery message $M \oplus \Delta M$ with the same NMAC value as the message $M$ in the related key case is given:

$$
\operatorname{Luffa}\left(K_{1}, \operatorname{Luffa}\left(K_{2}, M\right)\right)=\operatorname{Luffa}\left(K_{1}, \operatorname{Luffa}\left(K_{2} \oplus \Delta I V, M \oplus \Delta M\right)\right) .
$$

Where $\Delta M$ and $\Delta I V$ satisfy $M I(\Delta I V, \Delta M)=0$.

\section{The Pseudo-Preimage Attack on Luffa}

For Luffa-256, given a hash value $Z_{0}$, the adversary can compute a pseudo-preimage with the following process. An example is shown in Table 2 with $Z_{0}=\mathbb{0}$.

1. Select $Y_{0}, Y_{1}$ arbitrary, and get $Y_{3}=Z_{0} \oplus Y_{0} \oplus Y_{1}$.

2. Compute $X_{0}=Q_{0}^{-1}\left(Y_{0}\right), X_{1}=Q_{1}^{-1}\left(Y_{1}\right), X_{2}=Q_{2}^{-1}\left(Y_{2}\right)$.

3. Because the message $M=\mathbb{0}$ for the blank iteration, the adversary can compute $M I^{-1}\left(X_{0}, X_{1}, X_{2}\right)$ as follows,

$$
\left(\begin{array}{l}
H_{0} \\
H_{1} \\
H_{2}
\end{array}\right)=\left(\begin{array}{l}
0 \times 3,0 \times 2,0 \times 2 \\
0 \times 2,0 \times 3,0 \times 2 \\
0 \times 2,0 \times 2,0 \times 3
\end{array}\right)^{-1} \circ\left(\begin{array}{l}
X_{0} \\
X_{1} \\
X_{2}
\end{array}\right) .
$$

4. For the chaining variables $H_{0}, H_{1}, H_{2}$, the adversary can obtain $X_{0}=Q_{0}^{-1}\left(H_{0}\right)$, $X_{1}=Q_{1}^{-1}\left(H_{1}\right), X_{2}=Q_{2}^{-1}\left(H_{2}\right)$.

5. For $\left(X_{0}, X_{1}, X_{2}\right)$, the adversary computers $\left(I V_{1}^{\prime}, I V_{2}^{\prime}, M\right)$ with the fixed $I V_{0}$ by the following equations,

$$
\left(\begin{array}{c}
I V_{1}^{\prime} \\
I V_{2}^{\prime} \\
M
\end{array}\right)=\left(\begin{array}{l}
0 \times 2,0 \times 2,0 \times 1 \\
0 \times 3,0 \times 2,0 \times 2 \\
0 x 2,0 \times 3,0 \times 4
\end{array}\right)^{-1} \circ\left(\begin{array}{c}
X_{0} \oplus\left(3 \circ I V_{0}\right) \\
X_{1} \oplus\left(2 \circ I V_{0}\right) \\
X_{2} \oplus\left(2 \circ I V_{0}\right)
\end{array}\right)
$$


6. Output $\left(I V_{0}, I V_{1}^{\prime}, I V_{2}^{\prime}, M\right)$ which is the pseudo-preimage of $Z_{0}$, i.e.,

$$
\text { Luffa-256 }\left(I V^{\prime}, M\right)=Z_{0} \text {. }
$$

There are $w-1256$-bit words changed of the initial value with $w$ 256-bit words.

For Luffa-384, the hash value consists of $Z_{0}$ cascaded with the 128 most significant bits of $Z_{1}$, and

$$
Z_{1}=Z_{1,0}\left\|Z_{1,1}\right\| Z_{1,2}\left\|Z_{1,3}\right\| Z_{1,4}\left\|Z_{1,5}\right\| Z_{1,6} \| Z_{1,7}
$$

where $Z_{1, i}$ for $0 \leq i<8$ are 32-bit words. The adversary randomly chooses $\left(H_{0}, H_{1}, H_{2}\right)$, and gets $H_{3}=H_{0} \oplus H_{1} \oplus H_{2} \oplus Z_{0}$, computes $Z_{1}^{\prime}$ using the finalization function.

If the equation $Z_{1,0}^{\prime}\left\|Z_{1,1}^{\prime}\right\| Z_{1,2}^{\prime}\left\|Z_{1,3}^{\prime}=Z_{1,0}\right\| Z_{1,1}\left\|Z_{1,2}\right\| Z_{1,3}$ holds, let $Y_{0}=H_{0}, Y_{1}=$ $H_{1}, Y_{2}=H_{2}$. The adversary can now compute $\left(I V_{0}^{\prime}, I V_{1}^{\prime}, I V_{2}^{\prime}, I V_{3}^{\prime}\right)$ and message $M_{0}$ which has the same hash value $Z_{0}\left\|Z_{1,0}\right\| Z_{1,1}\left\|Z_{1,2}\right\| Z_{1,3}$, using the similar method with Luffa256 . The total complexity is $2^{128}$ iteration computations.

For Luffa-512, the complexity is $2^{255}$ using a similar attack.

\section{Improved Pseudo-Preimage Attacks on Luffa-384/512}

In this section, we introduce an algorithm to improve the pseudo-preimage attack on Luffa-384/512 by the extended generalized birthday attack which is used to solve a system of equations, proposed by Schnorr [6]. The $k$-dimensional generalization of the birthday problem is, given $k$ lists $L_{0}, L_{1}, \ldots, L_{k-1}$ independently at random from $\{0,1\}^{n}$, to find $k$ elements $x_{i} \in L_{i}$ for $0 \leq i \leq k-1$ such that $x_{0} \oplus x_{1} \oplus \cdots \oplus x_{k-1}=0$. Wagner's algorithm [7] builds a binary tree starting from the input lists $L_{0}, L_{1}, \ldots, L_{k-1}$. The time complexity and data complexity are both $t \cdot 2^{\frac{n}{1+t}}$, where $t=\left\lfloor\log _{2} k\right\rfloor$.

\subsection{The Extended Generalized Birthday Attack}

We give a brief description of Wagner's generalized birthday attack in the following.

Wagner's Algorithm.

1. The adversary constructs $2^{t}$ sets $S_{0}^{0}, S_{1}^{0}, \ldots, S_{2^{t}-1}^{0}$, where $t=\left\lfloor\log _{2} k\right\rfloor, S_{i}^{0}=\left\{x_{i}^{j}\right\rfloor$ $\left.x_{i}^{j} \in L_{i}, j=0,1, \ldots, 2^{\frac{n}{1+t}}-1\right\}$ for $1 \leq i<2^{t}-1$ and $S_{2^{t}-1}^{0}=\left\{x_{2^{t}-1}^{j} \oplus x_{2^{t}} \oplus \cdots \oplus x_{k} \mid\right.$ $\left.x_{2^{t}-1}^{j} \in L_{2^{t}-1}, j=0,1, \ldots, 2^{\frac{n}{1+t}}-1\right\}$, where $x_{l} \in L_{l}$ for $l=2^{t}, \ldots, k-1$.

2. The adversary searches $2^{\frac{n}{1+t}}$ element pairs $x_{2 i}^{j} \in S_{2 i}^{0}, x_{2 i+1}^{k} \in S_{2 i+1}^{0}$ with the same low $\frac{n}{1+t}$ bits by the birthday attack. Construct $2^{t-1}$ new sets $S_{i}^{1}, i=0,1, \ldots, 2^{t-1}-1$, where $S_{i}^{1}=\left\{x_{2 i}^{j} \oplus x_{2 i+1}^{k} \mid\right.$ the low $\frac{n}{1+t}$ bits are zeros $\}$.

3. For $m=2$ to $t-1$, the adversary searches $2^{\frac{n}{1+t}}$ pairs $x_{2 i}^{j} \in S_{2 i}^{m-1}$ and $x_{2 i+1}^{k} \in S_{2 i+1}^{m-1}$ with the $m$-th low $\frac{n}{1+t}$ bits same. Construct $2^{t-m}$ new sets $S_{i}^{m}, i=0,1, \ldots, 2^{t-m}-1$, where $S_{i}^{m}=\left\{x_{2 i}^{j} \oplus x_{2 i+1}^{k} \mid\right.$ the low $m \cdot \frac{n}{1+t}$ bits are zeros $\}$.

4. The adversary searches a pair $x_{0}^{j} \in S_{0}^{t-1}, x_{1}^{k} \in S_{1}^{t-1}$, s.t. $x_{0}^{j} \oplus x_{1}^{k}=0$. 
The above algorithm can find one solution $x_{0}, x_{1}, \ldots, x_{k-1}$ such that $x_{0} \oplus x_{1} \cdots \oplus x_{k-1}=0$ with time complexity and data complexity being both $t \cdot 2^{\frac{n}{1+t}}$.

Now consider the solution to the following two equations instead of one equation.

$$
\begin{aligned}
& f_{1}\left(x_{1}\right) \oplus f_{2}\left(x_{2}\right) \oplus \cdots \oplus f_{k}\left(x_{k}\right)=c_{1}, \\
& g_{1}\left(x_{1}\right) \oplus g_{2}\left(x_{2}\right) \oplus \cdots \oplus g_{k}\left(x_{k}\right)=c_{2},
\end{aligned}
$$

where $f_{i}$ and $g_{i}(1 \leq i \leq k)$ are random functions, $f_{i}: 2^{m} \rightarrow 2^{n_{1}}, g_{i}: 2^{m} \rightarrow 2^{n_{2}}$. The equations (1) and (2) can be solved together by the extended generalized birthday attack [6] described in the following.

It is easy to construct the following equation from equations (1) and (2):

$$
\left(f_{1}\left(x_{1}\right) \| g_{1}\left(x_{1}\right)\right) \oplus\left(f_{2}\left(x_{2}\right) \| g_{2}\left(x_{2}\right)\right) \oplus \cdots \oplus\left(f_{k}\left(x_{k}\right) \| g_{k}\left(x_{k}\right)\right)=c_{1} \| c_{2} .
$$

For the new equation (3), the Wagner's algorithm can be applied to obtain $x_{1}, \ldots, x_{k}$. The data and time complexity is $t \cdot 2^{\frac{n_{1}+n_{2}}{1+t}}$, where $t=\left\lfloor\log _{2} k\right\rfloor$ and $m \geq \frac{n_{1}+n_{2}}{1+t}$.

It is clear that, the algorithm can be extended to solve more equations.

$$
\begin{gathered}
f_{1}^{(1)}\left(x_{1}\right) \oplus f_{2}^{(1)}\left(x_{2}\right) \oplus \cdots \oplus f_{k}^{(1)}\left(x_{k}\right)=c_{1}, \\
f_{1}^{(2)}\left(x_{1}\right) \oplus f_{2}^{(2)}\left(x_{2}\right) \oplus \cdots \oplus f_{k}^{(2)}\left(x_{k}\right)=c_{2}, \\
\vdots \\
f_{1}^{(l)}\left(x_{1}\right) \oplus f_{2}^{(l)}\left(x_{2}\right) \oplus \cdots \oplus f_{k}^{(l)}\left(x_{k}\right)=c_{l},
\end{gathered}
$$

where $f_{j}^{(i)}: 2^{m} \rightarrow 2^{n_{i}}$ are random functions, $0 \leq i \leq l$, and $0 \leq j \leq k$. The data and time complexity is $t \cdot 2^{\frac{n_{1}+n_{2}+\cdots+n_{t}}{1+t}}$, where $t=\left\lfloor\log _{2} k\right\rfloor$ and $m \geq \frac{n_{1}+n_{2}+\cdots+n_{t}}{1+t}$.

\subsection{The Improved Pseudo-Preimage Attack on Luffa-384}

Let $\left(H_{0}, H_{1}, H_{2}, H_{3}, \mathbb{O}\right)$ be the input of the last blank iteration function, and $\left(X_{0}, X_{1}, X_{2}, X_{3}\right)$ be the output of its $M I$. The hash value is $Z_{0} \| \bar{Z}_{1}$, where $\bar{Z}_{1}=Z_{1,0}\left\|Z_{1,1}\right\| Z_{1,2} \| Z_{1,3}$. Then

$$
h_{128}\left(Q_{0}\left(X_{0}\right) \oplus Q_{1}\left(X_{1}\right) \oplus Q_{2}\left(X_{2}\right) \oplus Q_{3}\left(X_{3}\right)\right)=\bar{Z}_{1} .
$$

From the message injection function $M I$, we know that $\left(H_{0}, H_{1}, H_{2}, H_{3}\right)^{T}=A_{4 \times 4}^{-1}\left(X_{0}\right.$, $\left.X_{1}, X_{2}, X_{3}\right)^{T}$, where $A_{4 \times 4}$ is the first 4 column vectors of the matrix $A_{4 \times 5}$, i.e.,

$$
A_{4 \times 4}=\left(\begin{array}{l}
0 x 4,0 x 6,0 x 6,0 x 7 \\
0 x 7,0 x 4,0 x 6,0 x 6 \\
0 x 6,0 x 7,0 x 4,0 x 6 \\
0 x 6,0 x 6,0 x 7,0 x 4
\end{array}\right) .
$$

It's inverse matrix is

$$
A_{4 \times 4}^{-1}=\left(\begin{array}{l}
0 \times 20,0 \times 43,0 x 84,0 \times 11 \\
0 x 11,0 \times 20,0 x 43,0 x 84 \\
0 x 84,0 \times 11,0 \times 20,0 x 43 \\
0 x 43,0 x 84,0 \times 11,0 \times 20
\end{array}\right)
$$


From $H_{0} \oplus H_{1} \oplus H_{2} \oplus H_{3}=Z_{0}$, we can prove that,

$$
X_{0} \oplus X_{1} \oplus X_{2} \oplus X_{3}=Z_{0}^{\prime},
$$

where $Z_{0}^{\prime}=0 \times 3 \circ Z_{0}$.

Obviously, it is necessary for us to find the solution $\left(X_{0}, X_{1}, X_{2}, X_{3}\right)$ to make equations (4) and (5) hold together. We search the solution by the extended generalized birthday attack and some specific properties of Luffa. The algorithm is as follows.

1. The adversary constructs four sets such that,

$$
\begin{aligned}
& S_{0}=\left\{X_{0} \mid X_{0} \in\{0,1\}^{n}, l_{192}\left(X_{0}\right)=c_{0}\right\}, \\
& S_{1}=\left\{X_{1} \mid X_{1} \in\{0,1\}^{n}, l_{192}\left(X_{1}\right)=c_{0} \oplus l_{192}\left(Z_{0}^{\prime}\right)\right\}, \\
& S_{2}=\left\{X_{2} \mid X_{2} \in\{0,1\}^{n}, l_{192}\left(X_{2}\right)=c_{1}\right\}, \\
& S_{3}=\left\{X_{3} \mid X_{3} \in\{0,1\}^{n}, l_{192}\left(X_{3}\right)=c_{1}\right\},
\end{aligned}
$$

where $c_{0}, c_{1}$ are two 192-bit constants, and each set includes $2^{64}$ elements. It is clear that,

$$
l_{192}\left(X_{0} \oplus X_{1} \oplus X_{2} \oplus X_{3}\right)=l_{192}\left(Z_{0}^{\prime}\right),
$$

where $X_{i} \in S_{i}$ for $0 \leq i \leq 3$.

2. The adversary searches the solution $\left(X_{0}, X_{1}, X_{2}, X_{3}\right)$ satisfying the following two equations by the extended generalized birthday attack.

$$
\begin{gathered}
h_{64}\left(X_{0} \oplus X_{1} \oplus X_{2} \oplus X_{3}\right)=h_{64}\left(Z_{0}^{\prime}\right), \\
h_{128}\left(Q_{0}\left(X_{0}\right) \oplus Q_{1}\left(X_{1}\right) \oplus Q_{2}\left(X_{2}\right) \oplus Q_{3}\left(X_{3}\right)\right)=\bar{Z}_{1},
\end{gathered}
$$

where $X_{i} \in S_{i}, i=0,1,2,3$. It is clear that, The solution $\left(X_{0}, X_{1}, X_{2}, X_{3}\right)$ guarantees the equations (4) and (5) hold together.

3. For $\left(X_{0}, X_{1}, X_{2}, X_{3}\right)$, the adversary can calculate $\left(I V_{0}, I V_{1}^{\prime}, I V_{2}^{\prime}, I V_{3}^{\prime}\right)$ and the message $M$, and get the pseudo-preimage using the similar pseudo-preimage attack on Luffa256.

There are $2^{64} Q_{0}, Q_{1}, Q_{2}, Q_{3}$ computations and $2^{64}$ table lookups in the above algorithm. So the total complexity is about $2^{64}$ iteration computations and $2^{67}$ bytes memory.

\subsection{The Improved Pseudo-Preimage Attack on Luffa-512}

For Luffa-512, let $\left(H_{0}, H_{1}, H_{2}, H_{3}, H_{4}, 0\right)$ be the input of the last blank iteration function, and $\left(X_{0}, X_{1}, X_{2}, X_{3}, X_{4}\right)$ be the output of $M I$. Then

$$
Q_{0}\left(X_{0}\right) \oplus Q_{1}\left(X_{1}\right) \oplus Q_{2}\left(X_{2}\right) \oplus Q_{3}\left(X_{3}\right) \oplus Q_{4}\left(X_{4}\right)=Z_{1} .
$$


For the message injection function $M I$, we know that, $\left(H_{0}, H_{1}, H_{2}, H_{3}, H_{4}\right)^{T}=A_{5 \times 5}^{-1}\left(X_{0}\right.$, $\left.X_{1}, X_{2}, X_{3}, X_{4}\right)^{T}$, where $A_{5 \times 5}$ is the first 5 column vectors of the matrix $A_{5 \times 6}$, i.e.,

$$
A_{5 \times 5}=\left(\begin{array}{c}
0 x f, 0 x 8,0 x a, 0 x a, 0 x 8 \\
0 x 8,0 x f, 0 x 8,0 x a, 0 x a \\
0 x a, 0 x 8,0 x f, 0 x 8,0 x a \\
0 x a, 0 x a, 0 x 8,0 x f, 0 x 8 \\
0 x 8,0 x a, 0 x a, 0 x 8,0 x f
\end{array}\right) .
$$

Its inverse matrix is

$$
A_{5 \times 5}^{-1}=\left(\begin{array}{l}
0 x c 7,0 x 8 b, 0 x f 4,0 x f 4,0 x 8 b \\
0 x 8 b, 0 x c 7,0 x 8 b, 0 x f 4,0 x f 4 \\
0 x f 4,0 x 8 b, 0 x c 7,0 x 8 b, 0 x f 4 \\
0 x f 4,0 x f 4,0 x 8 b, 0 x c 7,0 x 8 b \\
0 x 8 b, 0 x f 4,0 x f 4,0 x 8 b, 0 x c 7
\end{array}\right) .
$$

Since $H_{0} \oplus H_{1} \oplus H_{2} \oplus H_{3} \oplus H_{4}=Z_{0}$, we obtain

$$
X_{0} \oplus X_{1} \oplus X_{2} \oplus X_{3} \oplus X_{4}=Z_{0}^{\prime},
$$

where $Z_{0}^{\prime}=0 x f \circ Z_{0}$.

We can search a solution to equations (6) and (7) by the extended generalized birthday attack and some specific properties of Luffa.

1. The adversary constructs four sets such that,

$$
\begin{aligned}
& S_{0}=\left\{X_{0} \mid X_{0} \in\{0,1\}^{n}, l_{128}\left(X_{0}\right)=c_{0}\right\}, \\
& S_{1}=\left\{X_{1} \mid X_{1} \in\{0,1\}^{n}, l_{128}\left(X_{1}\right)=c_{0} \oplus l_{128}\left(Z_{0}^{\prime}\right)\right\}, \\
& S_{2}=\left\{X_{2} \mid X_{2} \in\{0,1\}^{n}, l_{128}\left(X_{2}\right)=c_{1}\right\} \\
& S_{3}=\left\{\left(X_{3}, X_{4}\right) \mid X_{3}, X_{4} \in\{0,1\}^{n}, l_{128}\left(X_{3} \oplus X_{4}\right)=c_{1}\right\},
\end{aligned}
$$

where $c_{0}, c_{1}$ are two 128-bit constants, and each set includes $2^{128}$ elements. It is clear that,

$$
l_{128}\left(X_{0} \oplus X_{1} \oplus X_{2} \oplus X_{3} \oplus X_{4}\right)=l_{128}\left(Z_{0}^{\prime}\right) .
$$

2. The adversary searches a solution $\left(X_{0}, X_{1}, X_{2}, X_{3}, X_{4}\right)$ satisfying the following two equations by the extended generalized birthday attack.

$$
\begin{gathered}
h_{128}\left(X_{0} \oplus X_{1} \oplus X_{2} \oplus X_{3} \oplus X_{4}\right)=h_{128}\left(Z_{0}^{\prime}\right), \\
Q_{0}\left(X_{0}\right) \oplus Q_{1}\left(X_{1}\right) \oplus Q_{2}\left(X_{2}\right) \oplus Q_{3}\left(X_{3}\right) \oplus Q_{4}\left(X_{4}\right)=\bar{Z}_{1},
\end{gathered}
$$

where $X_{i} \in S_{i}, i=0,1,2$ and $\left(X_{3}, X_{4}\right) \in S_{3}$. It is clear that, The solution $\left(X_{0}, X_{1}, X_{2}, X_{3}, X_{4}\right)$ guarantees equations (6) and (7) hold.

3. For $\left(X_{0}, X_{1}, X_{2}, X_{3}, X_{4}\right)$, the adversary can calculate $\left(I V_{0}, I V_{1}^{\prime}, I V_{2}^{\prime}, I V_{3}^{\prime}, I V_{4}^{\prime}\right)$ and the message $M$, and get the pseudo-preimage using the similar pseudo-preimage attack on Luffa-256.

The total complexity is about $2^{128}$ iteration computations and $2^{132}$ bytes memory. 


\section{Conclusion}

In this paper, we give pseudo-collision, pseudo-second-preimage and pseudo-preimage attacks on Luffa, one of the second round candidates of SHA-3. For any given output of the message injection function $M I$, it is easy to get the input to $M I$ using the inverse operation of $M I$. So we can find pseduo-collisions and pseudo-second-preimages easily for Luffa by applying the $M I$ property. It is noted that, the pseudo-collisions and pseudosecond-preimages only with a few different bits are easily searched. The attack can be directly converted to a forgery attack on NMAC with related keys.

Especially, we focus on the the pseudo-preimage attack on Luffa. For Luffa-224/256, the attack can find the the pseudo-preimage only with 2 iteration computations. It takes about $2^{64}$ iteration computations and $2^{67}$ bytes memory to search a pseudo-preimage for Luffa-384, and search a pseudo-preimage with $2^{128}$ iteration computations and $2^{132}$ bytes memory for Luffa-512 with the extended generalized birthday attack.

Acknowledgements. We would like to thank the anonymous reviewers for their valuable comments on this paper. This work is supported by the National "973" Program of China (Grant No.2007CB807902) and Tsinghua University Initiative Scientific Research Program (2009THZ01002). For the second author, a part of this work was done while funded by EPSRC EP/C538285/1, by BT(as BT Chair of Information Security) and while visiting Tsinghua University funded by $2007 \mathrm{CB} 807902$.

\section{References}

1. Bellare, M., Canetti R., Krawczyk H.: Keying Hash Functions for Message Authentication. In: Koblitz, N. (ed.) CRYPTO 1996. LNCS, vol. 1109, pp. 1-15. Springer, Heidelberg (1996)

2. Cannière, C. D., Sato, H., Watanabe, D.: Hash function Luffa Specification. http://csrc.nist.gov/groups/ST/hash/sha-3/Round1/documents/Luffa.zip. (2008)

3. Contini, S., Yin, Y. L.: Forgery and Partial Key-Recovery Attacks on HMAC and NMAC Using Hash Collisions. In: Lai, X., Chen, K. (eds.): ASIACRYPT 2006. LNCS, vol. 4284, pp. 37-53. Springer, Heidelberg (2006)

4. Fouque, P.-A., Leurent, G., Nguyen, P. Q.: Full Key-Recovery Attacks on HMAC/NMACMD4 and NMAC-MD5. In: Menezes, A. (ed.) CRYPTO 2007. LNCS, vol. 4622, pp. 13-30. Springer, Heidelberg (2007)

5. Kelsey, J., Schneier, B.: Second Preimages on $n$-Bit Hash Functions for Much Less than $2^{n}$ Work. In: Cramer, R. (ed.): EUROCRYPT 2005, LNCS, vol. 3494, pp. 474-490. Springer, Heidelberg (2005)

6. Schnorr, C.P., Enhancing the Security of Perfect Blind DL-Signatures. Information Sciences. 176(i10), pp.1305-1320 (2006)

7. Wagner, D.: A generalized birthday problem. In: Yung, M. (ed.): CRYPTO 2002, LNCS, vol 2442, pp. 288-304. Springer, Heidelberg (2002)

8. Wang, X., Lai, X., Feng, D. et al.: Cryptanalysis of the hash functions MD4 and RIPEMD. In: Cramer, R. (ed.): Eurocrypt 2005, LNCS, vol 3494, pp. 1-18. Springer, Heidelberg (2005)

9. Wang, X., Yu, H., Yin, Y. L.: Efficient collision search attacks on SHA-0. In: Shoup, V. (ed.): CRYPT 2005, LNCS, vol 3621, pp. 1-16. Springer, Heidelberg (2005)

10. Wang, X., Yin, Y. L., Yu, H.: Finding collisions in the full SHA-1. In: Shoup, V. (ed.): CRYPT 2005, LNCS, vol 3621, pp. 17-36. Springer, Heidelberg (2005) 
11. Wang, X., Yu, H.: How to break MD5 and other hash functions. In: Cramer, R. (ed.): Eurocrypt 2005, LNCS, vol 3494, pp. 19-35. Springer, Heidelberg (2005)

12. Yu, H., Wang, G., Zhang, G. et al.: The second-preimage attack on MD4. In: Desmedt, Y. et al. (eds.): CANS 2005, LNCS, vol 3810, pp. 1-12. Springer, Heidelberg (2005)

\section{Appendix}

In the appendix,we give two examples for the pseudo-second-preimage and pseudopreimage.

\begin{tabular}{|l|llllll}
\hline$I V_{0}$ & 0x6d251e69 0x44b051e0 0x4eaa6fb4 & 0xdbf78465 0x6e292011 0x90152df4 0xee058139 0xdef610bb \\
\hline
\end{tabular} \begin{tabular}{l|llllllll}
\hline$V_{1}$ & 0xc3b44b95 0xd9d2f256 & 0x70eee9a0 & 0xde099fa3 & 0x5d9b0557 & 0x8fc944b3 & 0xcf1ccf0e & 0x746cd581 \\
\hline
\end{tabular}

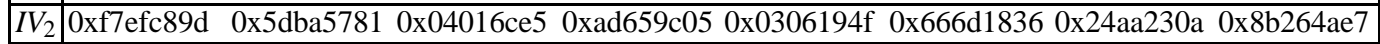

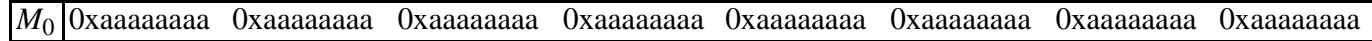

$I V_{0}^{\prime}$ 0x6d251e68 0x44b051e0 0x4eaa6fb5 $0 x d b f 78464$ 0x6e292011 0x90152df4 0xee058139 0xdef610bb

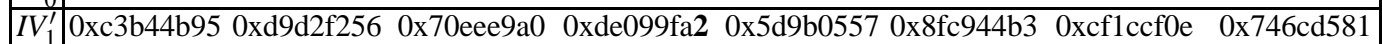

\begin{tabular}{|l|llllll}
\hline$I V_{2}^{\prime}$ & 0xf7efc89d & 0x5dba5780 & 0x4016ce5 & 0xad659c05 0x306194f & 0x666d1836 0x24aa230a 0x8b264ae7 \\
\hline
\end{tabular}

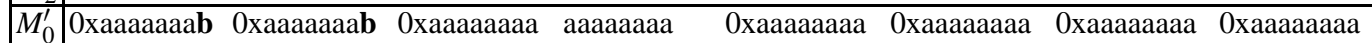

$X_{0}$ 0xe6333b1e 0x96d8e9f6 0x24d83129 0x6aa44be3 0x4da482a5 0x0a0bbb57 0x3d1e5ae2 0x71efd72c

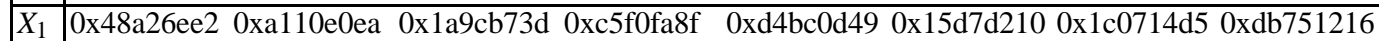

\begin{tabular}{|l|lllllll}
\hline$X_{2}$ & 0x7cf9edea & 0x2578453d 0xc4d998d2 & 0xb69cf929 $0 x 208 b b b f b$ & $0 x 56 d 9243 f$ & $0 x f 7 b 1 f 8 d 1$ & 0x243f8d70 \\
\hline
\end{tabular}

Table 1. A Pseudo-second-preimage for Luffa-256

\begin{tabular}{|l|llllll}
\hline$V_{0}$ & 0x6d251e69 & 0x44b051e0 0x4eaa6fb4 & 0xdbf78465 0x6e292011 0x90152df4 0xee058139 0xdef610bb \\
\hline
\end{tabular} $I V_{1}^{\prime} \mid$ 0x6a366118 0x3ee79df6 $0 x 39643181$ 0x60793777 0x8ddc9066 0x1d50cebd 0xb1cfd39b 0x967da4e4 $I V_{2}^{\prime}$ 0x9622ac99 0xb752bbbb 0xd256db58 0x73db6cac 0x9ae49b27 0xeb1666b4 0x805027ed 0x8176bfc6 $M$ 0x7c08aa09 $0 x 52 f 9 e 2 b f \quad$ 0x27ce6bb9 0x11af8970 $0 x 22 c 8478 d$ 0x9eebde0e 0x78ae77ef 0xdafc7fa8 $H_{0}$ 0xd42f102f $0 x 94316735$ 0xec5bb8a2 $0 x$ 0xceb338ee $0 x 6 \mathrm{~d} 35036 \mathrm{f}$ 0x85d4ba8c 0xc9a85c96 0xed839a52 \begin{tabular}{l|llllll}
\hline$H_{1}$ & 0x70238338 0x4461e9a7 0xa3012529 0xb6a10e0f $0 x d f d f 5 b c 0$ & 0x2fd50d38 0xe98ddd20 0xf90f4fe9
\end{tabular} \begin{tabular}{l|lllll}
$\mathrm{H}_{2}$ & 0xe0d87b07 0x5704423f $0 x b 8 b a 00 e d ~ 0 x e a a 52759$ & 0x8bc1b72b 0xc5720d53 0x41cde665 0x1288c8fc \\
\hline
\end{tabular}

\begin{tabular}{|l|llllllll|}
\hline$Z_{0}$ & 0 & 0 & 0 & 0 & 0 & 0 & 0 & 0 \\
\hline
\end{tabular}

Table 2. A Pseudo-preimage for Luffa-256 